\title{
Telemedicine-based diabetic retinopathy screening programs: an evaluation of utility and cost-effectiveness
}

This article was published in the following Dove Press journal:

Smart Homecare Technology and TeleHealth

10 June 2015

Number of times this article has been viewed

\section{Jorge A Cuadros}

Optometry/Vision Science, University of California, Berkeley, CA, USA
Correspondence: Jorge A Cuadros Optometry/Vision Science, University of California, Minor Hall I I0C, Berkeley, CA 94720, USA

Tel +I 5106425456

Email jcuadros@berkeley.edu
Abstract: Diabetes is the main cause of blindness among working age adults, although treatment is highly effective in preventing vision loss. Eye examinations are recommended on a yearly basis for most patients for timely detection of retinal disease. Telemedicine-based diabetic retinopathy screening (TMDRS) programs have been developed to identify patients with sightthreatening diabetic eye disease because patients are often noncompliant with recommended live eye examinations. This article reviews the cost-effectiveness of the various forms of TMDRS. A review of relevant articles, mostly published since 2008 , shows that societal benefits generally outweigh the costs of TMDRS. However, advances in technology to improve efficacy, lower costs, and broaden screening to other sight-threatening conditions, such as glaucoma and refractive error, are necessary to improve the sustainability of TMDRS within health care organizations. Patient satisfaction with these telemedicine programs is generally high. New models of shared care with primary care providers and staff are emerging to improve patient engagement and follow-up care when individuals are found to have sight-threatening eye disease. TMDRS programs are growing and provide valuable clinical benefit. The cost-utility is currently well proven in locations with limited access to regular eye care services, such as rural areas, poor communities, and prison systems; however, improvements over time are necessary for these programs to be cost-effective in mainstream medical settings in the future.

Keywords: telemedicine, diabetes, retinopathy, retinal imaging

\section{Rationale and description of TMDRS screening}

Diabetes is the leading cause of blindness among working age adults, and is estimated to be the cause of blindness in $4.8 \%$ of the 37 million people who are blind throughout the world, ${ }^{1}$ costing nearly \$US 5 billion per year in the USA alone. ${ }^{2}$ Vision impairment is largely preventable with early detection and treatment. ${ }^{3,4}$ While treatments are highly effective for preventing blindness, a large portion of patients with diabetes do not receive timely retinal examinations, which are recommended once per year for most patients. ${ }^{5}$ What is worse is that those individuals who are at most risk of developing severe retinal disease are often less likely to comply with recommended retinal examinations. For example, Sheppler et al found that poor glycemic control was significantly correlated with a reduced likelihood of compliance with retinal examinations, ${ }^{6}$ while Scanlon et al found that worse compliance with retinal examinations was significantly correlated with worse retinal disease at the time of examination. ${ }^{7}$ The low rates of compliance with traditional face-to-face eye examinations have prompted numerous telemedicine-based diabetic eye disease screening programs in primary care clinics, diabetes clinics, and community centers throughout 
the world. ${ }^{8}$ Store-and-forward telemedicine is used to bring remote ophthalmic expertise to diabetic patients in their primary health care environment in order to opportunistically identify individuals at risk of vision loss. ${ }^{9}$ Programs such as the US Veterans Administration program with more than 1 million encounters ${ }^{10}$ and the UK National Health Service Program that screens nearly 2 million people with diabetes per year, ${ }^{11}$ are examples of well-established programs in organizations that have committed considerable resources to continue their use of telemedicine for the foreseeable future. Hundreds of similar programs now exist throughout the world, with differing strategies depending on their settings, populations, and economic resources. As more organizations consider telemedicine/telehealth for detecting diabetic eye disease, questions arise regarding the costutility and return on investment (ROI) for their particular setting. In this article, we review the current technologies, screening strategies, and discuss the general cost-utility considerations of programs for detecting sight-threatening diabetic eye disease.

Uncontrolled diabetes causes microvascular changes in the retina leading to diabetic retinal lesions categorized as diabetic retinopathy (DR). The prevalence of any DR in the most recent studies of patients with diabetes is roughly $20 \%-40 \%{ }^{12-13}$ Chronic uncontrolled diabetes can eventually result in permanent vision loss, if not treated in a timely manner. ${ }^{14}$ Worsening DR leads to proliferative DR and clinically significant macular edema (CSME), which are the principal causes of permanent vision impairment in patients with diabetes. Proliferative DR is the growth of abnormal new blood vessels in the retina, and can cause retinal detachment and complete permanent blindness if unchecked. Treatment for proliferative DR is reported to be up to $90 \%$ effective in preventing blindness, if done in a timely manner. ${ }^{15} \mathrm{CSME}$ may cause permanent central blindness and occurs when leakage in the central retina disrupts photoreceptors. Timely treatment of CSME also dramatically reduces vision loss by up to $60 \%,{ }^{16,17}$ with greater efficacy using intravitreal injections of corticosteroids and anti-vascular endothelial growth factor medications. ${ }^{18}$ The proportion of vision impairment caused by CSME versus proliferative DR has been reported to be approximately 2 to 1 , but may vary depending on the population being screened..$^{19}$ The proportion of CSME and proliferative DR affects the strategy, techniques, and cost of detecting DR, depending on whether to emphasize viewing of the central or peripheral retina. ${ }^{20-22}$

The cost-effectiveness of telemedicine-based diabetic retinopathy screening (TMDRS) is well documented, with studies repeatedly concluding that this approach results in improved access and substantial savings compared with traditional methods of DR screening. ${ }^{24-26}$ Generally, the cost-effectiveness of TMDRS can be analyzed from three perspectives: the patient's perspective, considering the cost of the service to the patient, including transportation and lost time from work, versus the perceived benefit and satisfaction with the screening compared with traditional face-to-face eye examinations with optometrists or ophthalmologists; the organization's perspective (health care organization and/or payer), where the concern may be the cost of providing the service in order to obtain valuable quality measures and savings from averted costs for treating complications; and society's cost of the service versus the cost of vision impairment in terms of lost productivity, increased social services, and disability benefits. Essentially, all of these three perspectives weigh the resources required to receive or provide the service against the cost of blindness or complications related to deferred care using traditional eye care services. Cost-utility is related, but different from ROI, which affects organizations and individuals who directly pay for the service and affects telemedicine providers who gain revenue from the service. The ROI calculation is essentially the business case for providing the service, ie, the added revenue or cost savings from deferred complications and unnecessary services, less the expense of providing the service. ROI for TMDRS has been difficult to justify except in vertically integrated health care systems (ie, systems that bear the cost of blindness and vision impairment, such as government health services or large health maintenance organizations). For example, a US safety net program reduced the cost of retinal screening by $\$$ US 24.38 per encounter, but realized a $47 \%$ negative ROI for their 4-year program due to lack of reimbursement for the service. ${ }^{27}$ Adequate reimbursement and incentives to produce a positive ROI have been elusive for primary care clinics with regard to providing TMDRS; however, many payers are changing policies to provide sustainable support for these programs in the near future. Even with the low or sometimes negative ROI for non-vertically integrated systems, an increasing number of clinics have adopted TMDRS because of the proven clinical merit of the service for identifying patients with sight-threatening disease and the clinic's own mission to serve their patients' needs.

\section{Societal benefit}

Several researchers have investigated the benefit of performing TMDRS to the society, weighing the potential benefits of averting vision loss and quality-adjusted life years saved 
against the costs of TMDRS. Frick et al found that home care expenditures were most affected by vision impairment, along with higher medical care expenditures, a greater number of informal care days, and a decrease in health utility. ${ }^{28}$ Among individuals with diabetes, even a relatively small change in vision (ie, two lines of letters on a standard eye chart) was associated with a substantial change in social function, mental health, dependency, and role limitations. ${ }^{29}$ Rein et al concluded that TMDRS was cost-effective compared with annual or biennial eye examinations in communities where access to eye care services was limited or if TMDRS could be used to reasonably detect other causes of vision impairment, such as macular degeneration, glaucoma, cataract, and uncorrected refractive error. ${ }^{30}$ TMDRS has been shown to increase access to diagnostic eye care services in communities with limited access to specialty care while reducing the cost of surveillance. ${ }^{31}$ The California Health Care Foundation determined a lifetime cost saving for the state's medical assistance program to support TMDRS of approximately \$US 2,500 per patient with diabetes. ${ }^{32}$

\section{Patient perspective}

Unlike other forms of telemedicine that provide a consult for a specific complaint or condition, TMDRS is generally performed for patients who have no visual complaints or known active eye disease. One of the major challenges to implementation lies in motivating patients and providers to perform the service without any tangible immediate benefit for most participants. ${ }^{33}$

Organizations that adopt TMDRS will consider patient satisfaction as part of their cost-effectiveness analysis either before or after implementing a program in order to ensure participation of a sufficient proportion of patients to cover the capital costs. Patient feedback is generally positive, although patients often do not understand the purpose of the screening. ${ }^{34}$ Comments from patients are often related to convenience, reduced cost, and being impressed with the "high tech" procedure. ${ }^{35}$ Some studies found no or a slight increase in subsequent visits to ophthalmology of patients found to have sight-threatening DR, highlighting the need for greater patient engagement and calling into question the ultimate goal of averting vision impairment and its associated cost benefit to society. For example, Quade found only $22.8 \%$ compliance with treatment among TMDRS participants who were found to have sight-threatening DR in four highly functioning community clinics. ${ }^{36}$ Others found that informing patients of their retinopathy status had the greatest effect on their overall satisfaction with TMDRS and their subsequent diabetes control. ${ }^{37}$ Clear communication with patients about the purpose and necessity of TMDRS along with reliable communication of the results of the encounter are essential to maximize patient satisfaction and the effectiveness of the screening program. Ultimate cost-utility of TMDRS to prevent vision impairment cannot be realized without the added cost of including patient and provider education in the process.

\section{Organizational perspective}

TMDRS is clearly validated for detection of sight-threatening DR and may also detect other conditions; however, it does not take the place of regular eye examinations. Increased uptake of live face-to-face eye examinations among patients who have had TMDRS has been reported in the Veterans Administration program. ${ }^{38}$ This may be considered an added cost to payers who cover routine eye care; however, it may also indicate an increased awareness among patients of their eye conditions. TMDRS is well validated for detection of DR, but is not yet considered sufficiently sensitive and specific for detection of other diseases, such as glaucoma or macular degeneration. Progress is ongoing to develop effective programs for detection of all causes of blindness, and clearly any view of the eyes is better than no examination of the eyes at all in underserved populations. Studies have also found that TMDRS can increase patients' compliance with glycemic control. For example, the US Veterans Administration found that patients who underwent TMDRS that included patient education about diabetic eye disease had lower glycated hemoglobin than those that did not participate in TMDRS. ${ }^{39}$ Perhaps the greatest benefit of TMDRS may be to avert treatment altogether by improving patient education, leading to better glycemic control. Evidence suggests that patient education and patient engagement with their own retinal images can have a profound effect on their behavior and compliance with chronic disease recommendations. ${ }^{40}$

\section{Technical considerations}

Most TMDRS programs consist of: an acquisition device, usually a digital retinal camera; electronic means for image and data archival and transmission; and a system grading retinal images by human readers and/or computer algorithms in order to provide a diagnosis and referral recommendations.

\section{Image acquisition}

The performance and affordability of retinal imaging devices has evolved considerably over the past 20 years. Most of the 
early TMDRS programs use standard non-mydriatic retinal cameras that did not require eye drops for pupil dilation in most patients. Studies report that adequate images can be acquired in roughly $80 \%-95 \%$ of patients. ${ }^{41,42}$ Capturing one to three retinal images per eye with these cameras compares favorably with film mydriatic retinal cameras used in the large population studies that are now considered the gold standard for DR detection and staging, including the Early Treatment of Diabetic Retinopathy Study (ETDRS). ${ }^{43}$ Many of these standard non-mydriatic retinal cameras are in use; however, newer, lower-cost instruments that often perform better are gradually replacing them. At the time of publication, over 50 commercially available retinal imaging devices for TMDRS have been counted, generally ranging in cost between \$US 10,000 and \$US 50,000, with many more devices in development throughout the world. New devices fall into a few general categories, as follows. Any or all of the retinal imaging modalities discussed below can be used for TMDRS, and costs will likely decrease over time, undoubtedly increasing the cost-utility of future TMDRS programs.

\section{Tabletop standard color retinal cameras}

The necessary resolution for dependable lesion detection using tabletop standard color retinal cameras has been reported to be approximately 5 megapixels for a rectangular 45-degree image, although others have reported lower required resolution. Excessive image resolution (greater than 8 megapixels) does not improve image quality because of optical limitations of the eye, and requires brighter light flash and more computing resources. ${ }^{44}$ Another innovation in tabletop cameras is robotic focus, alignment, and light exposure. While many users appreciate the ease of operation and low learning curve, not all programs have used them successfully in their diabetic populations because they require greater cooperation and stability from their patients. Ogunyemi et al found that the time saved in training photographers to use robotic cameras was offset by the increased time required to capture adequate retinal images. ${ }^{45}$

\section{Scanning multiwave laser ophthalmoscopy}

Scanning multiwave laser ophthalmoscopy can generate high-resolution color images through smaller pupils and can sometimes capture a wider view of the retina. Although currently more expensive than standard retinal cameras, scanning laser imaging provides a promising and rapidly developing modality for image acquisition, reducing the image acquisition costs. ${ }^{46}$

\section{Optical coherence tomography}

Optical coherence tomography is also emerging as a TMDRS modality for detecting abnormalities of the central retina, particularly CSME. The sensitivity of optical coherence tomography to detect CSME is unsurpassed by any device, although the cost is typically higher than for standard retinal cameras.

\section{Handheld and smartphone retinal cameras}

Handheld and smartphone retinal cameras have received considerable attention in the public media, given that the dramatic affordability and portability of these devices is often presented as a great innovation for underserved populations. ${ }^{47}$ However, the difficulty in acquiring adequate images with these devices has kept them from being used in high-throughput, validated TMDRS programs. These devices will undoubtedly improve over time.

\section{Software for archival and transmission of images and data}

TMDRS is almost entirely deployed as a store-and-forward telemedicine application in order to maintain the affordability and adaptability of these programs. Diverse programs have evolved, with a large range of costs and features. Some programs have been developed "in-house" and may be little more than secure email and spreadsheets, while other programs have elaborate clinical and information technology protocols for complete, consistent, and secure communication. The cost and complexity of such a system is generally dictated by the policies of the organization and the goals of the screening program. For example, a hospital-based diabetes clinic that runs clinical trials would likely require stringent data collection protocols and high security within the network. Furthermore, a hospital may require that the TMDRS system interfaces with their electronic health records and/or picture archive communication system (PACS). Many configurations of these systems have been reported in the literature, and generally contain one or more of the following features:

- A mechanism for uploading images in an appropriate image file format, for example, DICOM, TIFF, or JPEG

- Structured data collection, including patient demographics and clinical history, eg, glycated hemoglobin, diabetes duration, and insulin dependence

- Unidirectional or bidirectional interfacing and data exchange with electronic health records or PACS

- Authentication and security, including disaster recovery and patient confidentiality measures 
- Reporting and alerts to inform clinicians about the need for reviewing images or to report that images have been reviewed

- Tracking referrals to ensure follow-up of patients who require further care

- Quality assurance through certification and credentialing of photographers, consultants, and other participating staff

- Administrative reporting tools for data analysis to understand trends and evaluate performance of the program.

The costs of these features vary greatly from free to several thousand \$US. In addition to the costs of the program and platform, the costs of implementation may be considerably greater. Most organizations who plan to implement TMDRS will calculate these costs; however, these costs are not published in the scientific literature.

\section{Image review and interpretation}

TMDRS programs are designed for retinal image interpretation to provide either a determination of presence or absence of retinal disease or a severity level of retinopathy. Systems may also provide referral and return recommendations, and may include notes about other eye conditions that are incidental to the DR grade (eg, comments about glaucoma and cataract). The American Telemedicine Association Ocular Telehealth Section has categorized screening programs into four levels of validation, as follows. ${ }^{48}$

Category 1 validation indicates that a system can separate patients into two categories, ie, those who have no or very mild nonproliferative DR (ETDRS level $\leq 20$ ), and those with levels of DR more severe than ETDRS level 20. Functionally, this level allows identification of patients who have no or minimal DR and those who have more than minimal DR.

Category 2 validation indicates that a system can accurately determine if sight-threatening DR is present or not present as evidenced by any level of diabetic macular edema, severe or worse levels of nonproliferative DR (ETDRS level $\geq 53$ ), or proliferative DR (ETDRS level $\geq 61$ ). This level allows identification of patients who do not have sight-threatening DR and those who do have potentially sight-threatening DR. Patients with sight-threatening DR generally require prompt referral for management.

Category 3 validation indicates that a system can identify ETDRS defined levels of nonproliferative DR (mild, moderate, or severe), proliferative DR (early, high-risk), and diabetic macular edema with accuracy sufficient to determine appropriate follow-up and treatment strategies. This level allows patient management to match clinical recommendations based on clinical retinal examination through dilated pupils. For example, it could give recommendations about the urgency of referral.

Category 4 validation indicates that a system matches or exceeds the ability of ETDRS photographs to identify DR lesions to determine levels of DR and macular edema. Functionally, category 4 validation indicates that a program can replace ETDRS photographs in any clinical or research program.

This level of validation as described by the American Telemedicine Association affects the cost of implementing the system, as well as the expected benefits and savings from the information and recommendations that they generate. Systems that simply detect the presence or absence of retinal pathology can be useful for organizations in which patients are generally healthy and only need to identify a few patients who would then be referred for a live eye examination. Computerized algorithms can often accomplish this level of grading, but must be fully validated to ensure a low rate of false negatives. These systems may also be a first pass to identify patients who require that their images be interpreted by human graders. Systems have been reported to reduce the human workload by $30 \%-70 \%$ by performing this first pass to eliminate patients with no apparent retinal pathology. ${ }^{49}$ Computer algorithms for automated image interpretation may offer a way to lower the cost of TMDRS by reducing the number of encounters for human interpretation of retinal images. They may also improve communication by offering real-time information for patient education and coordination of care. Image reading algorithms must be tested against images that are acquired in actual screening settings, since lower image quality can greatly diminish their performance. Validation against the standard retinal image databases, such as STARE, DRIVE, or MESSIDOR, shows sensitivity and specificity above $90 \%$ for most algorithms, ${ }^{50}$ however, they may not reflect performance in "real-world" settings. Image quality in actual screening settings is often less than optimal due to variability of photographers, reduced physical steadiness of patients, and smaller pupil size and cataracts, which occur with diabetes and advanced age. Future validation studies in actual screening settings are needed. Several strategies have emerged for human retinal image interpretation, and are discussed in the following paragraphs.

Reading centers often used trained non-medical personnel to grade retinal images for the presence and severity of diabetic retinal lesions. This approach has been used in largescale programs where the emphasis is on consistent quality of grading. Referral recommendations are standardized and not subject to nuances of clinical expertise. The consistency works well for clinical trials and large institutions; however, the nuanced clinical expertise is often valued by primary 
care clinics where patients can benefit from expert opinions about conditions that do not fit neatly into categories, such as pigment changes in the retina or subtle optic nerve findings. The theoretical cost savings of non-physician graders, as described by Bhargava et al, ${ }^{51}$ have yet to be realized.

Retinal image reading by ophthalmologists or optometrists experienced in retinopathy can offer greater expertise and more relevant referral and clinical recommendations than lay readers because eye care providers can comment on subtle and nuanced clinical findings. Organizations often look to their local eye care providers to perform TMDRS; however, reconciling ethical issues of self-referrals and maintaining their reputation in their communities often affects the viability of using local eye care providers for TMDRS and may reduce the cost-benefit if a very large number of patients screened are then referred for unnecessary examinations. Great variability in accuracy, consistency, and turnaround time has been reported in programs that use local eye care providers to read images. ${ }^{52}$ It is recommended that all readers undergo certification to orient them to the grading system and quality assurance by peer review to ensure consistency with grading.

Telementoring programs have been developed using telemedicine and remote connections to gradually guide primary care providers and other medical staff to interpret their own patients' retinal images. Most medical training programs include brief training of all medical residents in the detection of DR. Some programs, such as the University of Colorado and Salud Family Health Centers, have shown that primary care providers can effectively read retinal images. ${ }^{53}$ The EyePACS retinal reading program has trained and certified primary care providers and clinical staff to read retinal images, and has found the trained clinicians to be as accurate as eye care providers in identifying the presence and severity of retinal lesions associated with diabetes. ${ }^{54} \mathrm{~A}$ blend of both primary care and eye care providers viewing high-resolution digital retinal images is quite feasible, and frequently aids in the medical management of systemic disease, since retinal biomarkers and risk factors of systemic disease are often clearly visible in the images. This "shared care" approach to TMDRS and retinal imaging in general may become more viable as primary care providers learn to use retinal images as they would an ophthalmoscope. Images become much easier to access at the point of care when digital retinal images are tightly integrated with electronic health records. Cost savings and better patient management may result from primary care providers reading their own images, although the ability to consult with eye care providers on complicated cases will likely continue to be necessary. Organizations with low turnover of medical staff may find this strategy not only the most cost-effective, but may benefit clinically from the greater understanding of patients' microvascular status.

\section{Integrating TMDRS with detection of other conditions}

TMDRS is highly validated and accepted worldwide for detection of DR. Cost-benefit studies have largely focused on prevention of vision impairment from diabetes; however, many programs report an added benefit of discovering other conditions, such as glaucoma, macular degeneration, cardiovascular disease, and neurological conditions, incident to the TMDRS encounter. An adequate cost-benefit has not yet been proven for other conditions; however, progress has been made recently to deal intelligently with incidental findings that cannot ethically be ignored.

Glaucoma is one of the main causes of permanent, preventable blindness, where approximately $50 \%$ of affected individuals are unaware they have the disease. Screening programs for glaucoma have largely proven ineffective, with low economic or health benefit. ${ }^{55}$ However, given that patients return for retinal screening encounters year after year, it is possible to detect changes in the appearance of the optic nerve over time. Retinal imaging programs are now designed to facilitate detection of change in the appearance of optic nerves and other ocular structures over time. The rationale for collecting these images in the primary care setting is that patients will more consistently visit their "medical home" than they would visit the same eye care professional. This strategy of observing change over time may also facilitate detection of macular degeneration, vascular changes associated with cardiovascular disease, optic neuropathies, pigmented lesions, and other conditions. More studies are necessary to determine the techniques and strategies for optimizing the use of teleretinal imaging for other conditions besides diabetes, and ultimately, the added cost-utility.

\section{Optimizing screening intervals}

The American Diabetes Association has developed guidelines (Table 1) for the purpose of determining the frequency of retinal examinations for diabetic patients. Recent studies have concluded that well-controlled patients without retinopathy do not require annual retinal screening, while high-risk patients (ie, those with poor glycemic control or those who become pregnant) may require more frequent monitoring. New programs have been designed to determine the optimum screening interval on a case-by-case basis, 
Table I Screening interval guidelines from the American Diabetes Association standards of medical care, 2014

\begin{tabular}{|c|c|c|}
\hline Diabetes type & $\begin{array}{l}\text { First retinal } \\
\text { examination }\end{array}$ & $\begin{array}{l}\text { Subsequent } \\
\text { examinations }\end{array}$ \\
\hline Type I diabetes & $\begin{array}{l}\text { Within } 5 \text { years of } \\
\text { diagnosis and after } \\
\text { onset of puberty }\end{array}$ & Annually \\
\hline Type 2 diabetes & At time of diagnosis & Annually \\
\hline $\begin{array}{l}\text { Type I and } 2 \text {, well controlled, } \\
\text { and no retinopathy on } \\
\text { previous eye examination }\end{array}$ & No change & Every 2 years \\
\hline $\begin{array}{l}\text { Type I and } 2 \text {, } \\
\text { with retinopathy }\end{array}$ & No change & $\begin{array}{l}\text { As recommended } \\
\text { by eye care } \\
\text { provider }\end{array}$ \\
\hline $\begin{array}{l}\text { Women with pre-existing } \\
\text { diabetes who become } \\
\text { pregnant }\end{array}$ & $\begin{array}{l}\text { During first } \\
\text { trimester }\end{array}$ & $\begin{array}{l}\text { Close follow-up } \\
\text { until I year } \\
\text { postpartum }\end{array}$ \\
\hline
\end{tabular}

customized for each diabetic patient. Cost savings of \$US 200 million have been projected for implementing these programs in the USA. ${ }^{56}$

\section{Conclusion}

Telemedicine-based DR screening has grown steadily worldwide during the past decade and has become well established in several large organizations. While clinicians continue to struggle with reimbursement from payers for the service, the clinical and societal benefits are well documented. Patients are generally satisfied with the service, but often do not understand the benefits or rationale for the service. It is crucial to incorporate effective patient communication in order to realize the full benefits of telemedicine for effective triage of sight-threatening conditions and for better systemic control of chronic disease. Local primary care providers involved in shared-care arrangements with remote eye care providers ("telementoring") can effectively increase compliance with treatments and lower the long-term costs of DR screening.

Technology for increasing the efficiency and effectiveness of TMDRS is evolving rapidly. Lower-cost imaging devices, electrodiagnostic devices, optical coherence tomography, integrated information systems, and automated algorithms for detection of retinal lesions are being used to expand access to TMDRS and to improve the precision of identifying who needs retinal screening and who needs referral for secondary and tertiary ophthalmic care. New telemedicine programs are in development for detection of macular degeneration, glaucoma, and other sight-threatening conditions. These new programs will be important in order to sustain the service in the future. Eye care telemedicine is here to stay, and the question is not whether it will be sustainable in the future, but what other eye care services will be added on to the networks that are forming now.

\section{Disclosure}

The author reports ownership in the EyePACS retinal reading program.

\section{References}

1. World Health Organization. Prevention of blindness from diabetes mellitus: report of a WHO Consultation. Geneva, Switzerland: World Health Organization; 2005. Available from: http://www.who.int/ blindness/Prevention $\% 20$ of $\% 20$ Blindness $\% 20$ from $\% 20$ Diabetes $\% 20$ Mellitus-with-cover-small.pdf. Accessed April 17, 2015.

2. Prevent Blindness America. The economic impact of vision problems, 2007. Available from: http://documents.preventblindness.org/ publichealth/Impact_of_Vision_Problems.pdf. Accessed January 20, 2015.

3. [No authors listed]. Treatment techniques and clinical guidelines for photocoagulation of diabetic macular edema. Early Treatment Diabetic Retinopathy Study Research Group. Ophthalmology. 1987;94: 761-774.

4. [No authors listed]. Photocoagulation treatment of proliferative diabetic retinopathy: clinical application of Diabetic Retinopathy Study (DRS) findings, DRS report no 8. Diabetic Retinopathy Study Research Group. Ophthalmology. 1981;88:583-600.

5. American Diabetes Association. Standards of medical care in diabetes2014. Diabetes Care. 2014;37 Suppl 1:S14-S80.

6. Sheppler CR, Lambert WE, Gardiner SK, Becker TM, Mansberger SL. Predicting adherence to diabetic eye examinations: development of compliance with annual diabetic eye exams survey. Ophthalmology. 2014;121:1212-1219.

7. Scanlon PH, Aldington SJ, Stratton IM. Delay in diabetic retinopathy screening increases the rate of detection of referable diabetic retinopathy. Diabet Med. 2014;31:439-442.

8. Yogesan K. Teleophthalmology. Berlin, Germany: Springer; 2006.

9. Yogesan K, Goldschmidt LP, Cuadros J. Digital Teleretinal Screening. Teleophthalmology in Practice. Berlin, Germany: Springer; 2012.

10. Cavallerano AA, Conlin PR. Teleretinal imaging to screen for diabetic retinopathy in the Veterans Health Administration. $J$ Diabetes Sci Technol. 2008;2:33-39.

11. Papavasileiou E, Dereklis D, Oikonomidis P, et al. An effective programme for systematic diabetic retinopathy screening in order to reduce diabetic retinopathy blindness. Hell J Nucl Med. 2014;17 Suppl 1: 30-34.

12. Yau JW, Rogers SL, Kawasaki R, et al; for the Meta-Analysis for Eye Disease (META-EYE) Study Group. Global prevalence and major risk factors of diabetic retinopathy. Diabetes Care. 2012;35: $556-564$.

13. Varma R, Bressler NM, Doan QV, et al. Prevalence of and risk factors for diabetic macular edema in the United States. JAMA Ophthalmol. 2014;132:1334-1340.

14. Schwartz S, Harasawa M, Baldivieso V, et al. Nonmydriatic fundus camera for diabetic retinopathy screening in a safety net hospital: effectiveness, prevalence, and risk factors. Eur J Ophthalmol. 2015;25: $145-152$.

15. Venkatesh P, Tibrewal S, Bhowmik D, et al. Prevalence of systemic co-morbidities in patients with various grades of diabetic retinopathy. Indian J Med Res. 2014;140:77-83.

16. Mathenge W, Bastawrous A, Peto T, et al. Prevalence and correlates of diabetic retinopathy in a population-based survey of older people in Nakuru, Kenya. Ophthalmic Epidemiol. 2014;21:169-177.

17. Liu L, Geng J, Wu J, et al. Prevalence of ocular fundus pathology with type 2 diabetes in a Chinese urban community as assessed by telescreening. BMJ Open. 2013;3:e004146. 
18. Klein R, Klein BE, Moss SE. The Wisconsin epidemiological study of diabetic retinopathy: a review. Diabetes Metab Rev. 1989;5:559-570.

19. Ferris FL. How effective are treatments for diabetic retinopathy? JAMA. 1993;269:1290-1291.

20. [No authors listed]. Photocoagulation for diabetic macular edema. Early Treatment Diabetic Retinopathy Study report number 1. Early Treatment Diabetic Retinopathy Study Research Group. Arch Ophthalmol. 1985;103:1796-1806.

21. [No authors listed]. Early Treatment Diabetic Retinopathy Study Research Group. Focal photocoagulation treatment of diabetic macular edema. Relationship of treatment effect to fluorescein angiographic and other retinal characteristics at baseline: ETDRS report no 19. Arch Ophthalmol. 1995;113:1144-1155.

22. Arevalo JF. Diabetic macular edema: current management 2013. World J Diabetes. 2013;4:231-233.

23. Moss SE, Klein R, Klein BE. The 14-year incidence of visual loss in a diabetic population. Ophthalmology. 1998;105:998-1003.

24. Jones S, Edwards RT. Diabetic retinopathy screening: a systematic review of the economic evidence. Diabet Med. 2010;27:249-256.

25. Gomez-Ulla F, Alonso F, Aibar B, Gonzalez F. A comparative cost analysis of digital fundus imaging and direct fundus examination for assessment of diabetic retinopathy. Telemed J E Health. 2008;14:912-918.

26. Rachapelle S, Legood R, Alavi Y, et al. The cost-utility of telemedicine to screen for diabetic retinopathy in India. Ophthalmology. 2013;120:566-573.

27. Phan AT, Koczman JJ, Yung C-W, Pernic AA, Doerr ED, Kaehr MM. Cost analysis of teleretinal screening for diabetic retinopathy in a county hospital population. Diabetes Care. 2014;37:e252-e253.

28. Frick KD, Gower EW, Kempen JH, et al. Economic impact of visual impairment and blindness in the United States. Arch Ophthalmol. 2007; 125:544-550.

29. Matza LS, Rousculp MD, Malley K, Boye KS, Oglesby A. The longitudinal link between visual acuity and health-related quality of life in patients with diabetic retinopathy. Health Qual Life Outcomes. 2008;6:95.

30. Rein DB, Wittenborn JS, Zhang X, et al. The cost-effectiveness of three screening alternatives for people with diabetes with no or early diabetic retinopathy. Health Serv Res. 2011;46:1534-1561.

31. Li Z, Wu C, Olayiwola JN, Hilaire DS, Huang JJ. Telemedicine-based digital retinal imaging vs standard ophthalmologic evaluation for the assessment of diabetic retinopathy. Conn Med. 2012;76:85-90.

32. Newman M. The longitudinal link between visual acuity and healthrelated quality of life in patients with diabetic retinopathy. Available from: http://www.chcf.org/resources/download.aspx?id=\%7b1335FD441579-4A69-B503-918E4414800B\%7d. Accessed January 22, 2015.

33. Hipwell AE, Sturt J, Lindenmeyer A, et al. Attitudes, access and anguish: a qualitative interview study of staff and patients' experiences of diabetic retinopathy screening. BMJ Open. 2014;4:e005498.

34. Orton E, Forbes-Haley A, Tunbridge L, Cohen S. Equity of uptake of a diabetic retinopathy screening programme in a geographically and socioeconomically diverse population. Public Health. 2013;127:814-821.

35. Newman R, Cummings DM, Doherty L, Patel NR. Digital retinal imaging in a residency-based patient-centered medical home. Fam Med. 2012;44:159-163.

36. Quade R. Evaluation of the expanding access to diabetic retinopathy screening initiative. Available from: http:/www.chcf.org/projects/2009/ diabetic-retinopathy-screening\#ixzz3PgTsJLGW. Accessed January 25, 2015.

37. García Serrano MJ, Asensi Blanch A, Farré Marimon JM, et al. [User satisfaction with teleophthalmology with nonmydriatic camera for diabetic retinopathy screening]. Gac Sanit. 2009;23:322-325. Spanish.

38. Conlin PR, Fisch BM, Cavallerano AA, et al. Nonmydriatic teleretinal imaging improves adherence to annual eye examinations in patients with diabetes. J Rehabil Res Dev. 2006;43:733-740.
39. Bursell SE, Brazionis L, Jenkins A. Telemedicine and ocular health in diabetes mellitus. Clin Exp Optom. 2012;95:311-327.

40. Rees G, Lamoureux EL, Nicolaou TE, et al. Feedback of personal retinal images appears to have a motivational impact in people with non-proliferative diabetic retinopathy and suboptimal $\mathrm{HbA}_{1 \mathrm{c}}$ : findings of a pilot study. Diabet Med. 2013;30:1122-1125.

41. Scanlon PH, Malhotra R, Thomas G, et al. The effectiveness of screening for diabetic retinopathy by digital imaging photography and technician ophthalmoscopy. Diabet Med. 2003;20:467-474.

42. Whited JD. Accuracy and reliability of teleophthalmology for diagnosing diabetic retinopathy and macular edema: a review of the literature. Diabetes Technol Ther. 2006;8:102-111.

43. Shi L, Wu H, Dong J, et al. Telemedicine for detecting diabetic retinopathy: a systematic review and meta-analysis. Br J Ophthalmol. January 6, 2015. [Epub ahead of print.]

44. Jensen PK, Scherfig E. Resolution of retinal digital colour images. Acta Ophthalmol Scand. 1999;77:526-529.

45. Ogunyemi O, Moran E, Patty Daskivich L, et al. Autonomy versus automation: perceptions of nonmydriatic camera choice for teleretinal screening in an urban safety net clinic. Telemed J E Health. 2013;19: 591-596.

46. Rasmussen ML, Broe R, Frydkjaer-Olsen U, et al. Comparison between Early Treatment Diabetic Retinopathy Study 7-field retinal photos and non-mydriatic, mydriatic and mydriatic steered widefield scanning laser ophthalmoscopy for assessment of diabetic retinopathy. J Diabetes Complications. 2015;29:99-104.

47. Russo A, Morescalchi F, Costagliola C, Delcassi L, Semeraro F. Comparison of smartphone ophthalmoscopy with slit-lamp biomicroscopy for grading diabetic retinopathy. Am J Ophthalmol. 2015;159: 360-364. e1.

48. Cavallerano J, Lawrence MG, Zimmer-Galler I, et al; American Telemedicine Association, Ocular Telehealth Special Interest Group; National Institute of Standards and Technology Working Group. Telehealth practice recommendations for diabetic retinopathy. Telemed $J$ E Health. 2004; 10:469-482.

49. Bernardes R, Serranho P, Lobo C. Digital ocular fundus imaging: a review. Ophthalmologica. 2011;226:161-181.

50. Kauppi T, Kämäräinen JK, Lensu L, et al. Constructing benchmark databases and protocols for medical image analysis: diabetic retinopathy. Comput Math Methods Med. 2013;2013:368514.

51. Bhargava M, Cheung CY, Sabanayagam C, et al. Accuracy of diabetic retinopathy screening by trained non-physician graders using nonmydriatic fundus camera. Singapore Med J. 2012;53:715-719.

52. Sellahewa L, Simpson C, Maharajan P, Duffy J, Idris I. Grader agreement, and sensitivity and specificity of digital photography in a community optometry-based diabetic eye screening program. Clin Ophthalmol. 2014;8:1345-1349.

53. Farley TF, Mandava N, Prall FR, Carsky C. Accuracy of primary care clinicians in screening for diabetic retinopathy using single-image retinal photography. Ann Fam Med. 2008;6:428-434.

54. Cuadros J, Bresnick G, Le J, See J. Knowledge transfer technology and tele-mentoring applied to primary care. Care providers review diabetic retinal images in real time, supported by remote experts. Poster presented at the Seventh Advanced Technologies and Treatments for Diabetes Annual Conference, March 28, 2013, Paris, France.

55. Hatt S, Wormald R, Burr J. Screening for prevention of optic nerve damage due to chronic open angle glaucoma. Cochrane Database Syst Rev. 2006;4:CD006129.

56. Leese GP, Stratton IM, Land M, et al. Progression of diabetes retinal status within community screening programs and potential implications for screening intervals. Diabetes Care. 2015;38:488-494. 
Smart Homecare Technology and TeleHealth

Dovepress

\section{Publish your work in this journal}

Smart Homecare Technology and TeleHealth is an international, peer-reviewed, open access online journal publishing original research, reviews, editorials and commentaries on the application of technology to support people and patients at home and in assisted living centers to optimize healthcare and management resources. Specific topics in the journal include: Development and application of

devices within the home and embedded in appliances; Healthcare provider communication and education tools; and drug ordering and adherence. The manuscript management system is completely online and includes a very quick and fair peer-review system, which is all easy to use. Visit http://www.dovepress.com/ testimonials.php to read real quotes from published authors.

Submit your manuscript here: http://www.dovepress.com/smart-homecare-technology-and-telehealth-journal 